\title{
Molten Globule Formation in Apomyoglobin Monitored by the Fluorescent Probe Nile Red ${ }^{\dagger}$
}

\author{
Eugenia Polverini, $\$$, Giuseppe Cugini, $\$, \S$ Francesca Annoni,,$\$$ Stefania Abbruzzetti,,$\S$ Cristiano Viappiani,,$\neq, \$$ and \\ Thomas Gensch" \\ *Dipartimento di Fisica, Università degli Studi di Parma, Viale G. P. Usberti 7/A, 43100 Parma, Italy, \\ CNR-INFM, Viale G. P. Usberti 7/A, 43100 Parma, Italy, and Forschungszentrum Jülich, \\ Institut für Biologische Informationsverarbeitung 1, 52425 Jülich, Germany
}

Received September 19, 2005; Revised Manuscript Received December 28, 2005

\begin{abstract}
The interaction of nile red (NR) with apomyoglobin (ApoMb) in the native (pH 7) and molten globule ( $\mathrm{pH} 4)$ states was investigated using experimental and computational methods. NR binds to hydrophobic locations in ApoMb with higher affinity $\left(K_{\mathrm{d}}=25 \pm 5 \mu \mathrm{M}\right)$ in the native state than in the molten globule state $\left(K_{\mathrm{d}}=52 \pm 5 \mu \mathrm{M}\right)$. In the molten globule state, NR is located in a more hydrophobic environment. The dye does not bind to the holoprotein, suggesting that the binding site is located at the heme pocket. In addition to monitoring steady-state properties, the fluorescence emission of NR is capable of tracking submillisecond, time-resolved structural rearrangements of the protein, induced by a nanosecond $\mathrm{pH}$ jump. Molecular dynamics simulations were run on $\mathrm{ApoMb}$ at neutral $\mathrm{pH}$ and at $\mathrm{pH} 4$. The structure obtained for the molten globule state is consistent with the experimentally available structural data. The docking of NR with the crystal structure shows that the ligand binds into the binding pocket of the heme group, with an orientation bringing the planar ring system of NR to overlap with the position of two of the heme porphyrin rings in $\mathrm{Mb}$. The docking of NR with the ApoMb structure at $\mathrm{pH} 4$ shows that the dye binds to the heme pocket with a slightly less favorable binding energy, in keeping with the experimental $K_{\mathrm{d}}$ value. Under these conditions, NR is positioned in a different orientation, reaching a more hydrophobic environment in agreement with the spectroscopic data.
\end{abstract}

Apomyoglobin $\left(\mathrm{ApoMb}^{1}\right)$, that is, myoglobin $(\mathrm{Mb})$ without the heme group, contains eight strands of mostly $\alpha$-helical segments, labeled $\mathrm{A}-\mathrm{H}$. ApoMb adopts, at neutral $\mathrm{pH}$, a structure $(N)$ that is similar to that of the native $\mathrm{Mb}$ according to the available NMR, $\mathrm{CD}$, and calorimetric evidence ( $1-$ 5). The ApoMb structure is characterized by a hydrophobic core, consisting at least of the very stable helices $\mathrm{A}, \mathrm{G}$, and $\mathrm{H}$, with a secondary structure and tertiary folding pattern similar to that of native $\mathrm{Mb}$. Although the $\mathrm{A}, \mathrm{G}$, and $\mathrm{H}$ helices form a distinct tight subdomain in $\mathrm{Mb}$, these helices are unstable when isolated as separate fragments $(6,7)$. At $\mathrm{pH} 4$, ApoMb adopts a conformation $\left(I_{4}\right)$ that has been the subject of numerous structural, thermodynamic, kinetic, and theoretical studies $(5,8,9)$. The $I_{4}$ conformation shows a decreased helix content and lacks the tight side-chain packing characteristic of the cores of globular proteins (6). The hydrogen bonding between His 24 and His119 located in helices B and G, respectively, is important for the integrity

\footnotetext{
$\dagger$ We acknowledge MIUR (FIRB nanotechnologies and PRIN2004) and CNR-INFM for financial funding.

* Corresponding author. Tel: +39 0521 905208. Fax: +390521 905223. E-mail: cristiano.viappiani@fis.unipr.it.

Dipartimento di Fisica, Parco Area delle Scienze 7/A.

$\S$ CNR-INFM, Parco Area delle Scienze 7/A.

"Institut für Biologische Informationsverarbeitung 1.

${ }^{1}$ Abbreviations: NR, nile red; ApoMb, apomyoglobin; Mb, myoglobin; $I$, molten globule state of apomyoglobin; $N$, native state of apomyoglobin; MD, molecular dynamics; 2-NBA, 2-nitrobenzaldehyde; rmsd, root-mean-square displacement; SASA, solvent-accessible surface area.
}

of the $N$ form (8). At $\mathrm{pH} 4$, this hydrogen bond is broken and the partial unfolding of helix $\mathrm{B}$ and the $C$-terminal of helix $\mathrm{G}$ is occurring.

When $\mathrm{ApoMb}$, unfolded by $\mathrm{GuHCl}$ or urea, is refolded at $\mathrm{pH} 7$, the formation of a folding intermediate in less than a millisecond has been proven by applying stopped-flow techniques, small-angle X-ray scattering, and pulsed hydrogen-deuterium exchange measurements $(10,11)$. This kinetically observed form $\left(I_{7}\right)$ shows strong similarities with the $I_{4}$ form trapped at low $\mathrm{pH}$, including the existence of helices $\mathrm{A}, \mathrm{G}$, and $\mathrm{H}$ and the pattern of protected amide $\mathrm{NH}$ groups of the protein backbone $(1,10)$. It is therefore likely that the species $I_{4}$ trapped at low $\mathrm{pH}$ represents the kinetic folding intermediate $I_{7}$ observed in refolding experiments at neutral $\mathrm{pH}$ and, thus, will be referred to as intermediate I herein. Its nature is identified as a molten globule from the various thermodynamic and secondary structure information. Molten globule states can be found as transient states in many folding and unfolding processes.

The refolding involves the motions of large portions of the molecule and is expected to proceed through a series of events characterized by different rates, ranging from nanoseconds to milliseconds. It has been suggested that the formation of the hydrogen bond between His24 (helix B) and His119 (helix G) may be a rate-limiting step in the refolding of intermediate $I_{7}$ to the native $N$ form (8). Investigation of the pressure induced formation of an $I$ form, similar to the one observed at low $\mathrm{pH}$ and in the kinetic 
folding pathway, has revealed that the volume of this species is considerably smaller $(\Delta V=-70 \mathrm{~mL} / \mathrm{mol})$ compared to that of the $N$ form (12). Recent time-resolved photoacoustic measurements using a nanosecond $\mathrm{pH}$ jump as the trigger for the unfolding reaction $N \rightarrow I$ showed that this huge contraction is produced within a few microseconds (13). This finding suggests that much of the structural change for the $N \rightarrow I$ transition, triggered by protonation of His 24 and His119, occurs on the same time scale. A probe beam deflection study recently proved that no additional structural volume changes are detectable up to millisecond time scales (14).

Nile red (NR) is a fluorescent molecule, which probes the polarity of its environment $(15,16)$. It shows large changes in fluorescence quantum yields and spectra due to changes in the polarity. NR has a low fluorescence quantum yield in a polar environment, whereas in more hydrophobic environments its quantum yield increases, and its emission maximum becomes progressively blue shifted. The low fluorescence emission in polar solvents has been attributed to the efficient formation of a twisted intramolecular charge transfer state $(15,17,18)$, which, in turn, efficiently forms a triplet state. The formation of the charge transfer state is inhibited in apolar solvents, and the rotation of the diethilamino group is prevented. Also, the molecule is stabilized in its highly fluorescent excited-singlet state.

NR has been used to identify hydrophobic sites exposed to the solution and conformational changes in a number of proteins, including calmodulin and albumin (19). In a recent study, it was also used to identify a transiently exposed site in the photoactive yellow protein from Ectothiorhodospira halophila (20).

In this work, we have investigated the interaction of NR with ApoMb at neutral and acidic $\mathrm{pH}(\mathrm{pH} 4)$ by means of experimental and computational techniques. Unlike previous computational studies dealing with very acidic unfolding conditions ( $\mathrm{pH} 2),(9)$ in this work, we have considered the protein at $\mathrm{pH} 4$ to characterize the molten globule state. At this intermediate $\mathrm{pH}$, the protonation state of the protein is very critical and must be accurately modeled, a problem that was neglected in previous investigations (21). We have carefully considered the protonation state of the protonatable amino acid residues of the protein and performed two molecular dynamics (MD) simulations in which the protonation state of ambiguous residues was considered in the protonated and deprotonated states. Docking simulations with NR were carried out on the structure of intermediate $I_{4}$ at pH 4 obtained by a MD simulation. We also performed a MD simulation on the ApoMb structure at neutral $\mathrm{pH}(\mathrm{pH}$ 7 ), and the retrieved structure was used to characterize the interaction with NR.

\section{MATERIALS AND METHODS}

Chemicals. 2-Nitrobenzaldehyde (2-NBA) was obtained from Sigma and was recrystallized from ethanol before use. Horse heart $\mathrm{Mb}$ was from Sigma. NR was purchased from Sigma-Aldrich (Munich, Germany).

Apomyoglobin Preparation. Apomyoglobin was prepared by cold $\left(-30{ }^{\circ} \mathrm{C}\right)$ acid-acetone extraction from horse heart $\mathrm{Mb}$ (22). The sample was washed with cold acetone, centrifuged several times, dried with pure nitrogen, and suspended in water at $\mathrm{pH}$ 7. The suspension was then centrifuged, and the supernatant was spectroscopically checked to assess sample purity. The concentration of the ApoMb stock was calculated from the absorption at $280 \mathrm{~nm}$ $\left(\epsilon=15800 \mathrm{~cm}^{-1} \mathrm{M}^{-1}\right)$, and heme contamination was estimated from the absorption at $408 \mathrm{~nm}(\epsilon=179000$ $\mathrm{cm}^{-1} \mathrm{M}^{-1}$ ) (23). In all preparations used, $99.5 \%$ of the total protein was free of the heme dye.

Steady-State Absorption, Far-UV Circular Dichroism and Steady-State Fluorescence Emission. Steady-state absorption was measured with a Jasco $7850 \mathrm{UV}$-vis spectrophotometer. The steady-state fluorescence emission $\left(\lambda_{\mathrm{ex}}=295 \mathrm{~nm}\right)$ was measured with a Perkin-Elmer LS50 spectrofluorometer. FarUV circular dichroism was measured with a Jasco J700. All instruments were equipped with temperature controlled sample holders. Fluorescence quantum yields were determined by a comparative method using $\mathrm{NR}$ in $\mathrm{H}_{2} \mathrm{O}\left(\Phi_{\mathrm{F}}=\right.$ $0.02(16))$ as a standard.

Determination of the Dissociation Constant. The dissociation constant was determined from the observed changes in fluorescence emission as a function of protein concentration. The observed changes were plotted in a double reciprocal plot, and the affinity was determined from the slope (24).

Nanosecond pH-Jump Setup. The nanosecond $\mathrm{pH}$ jump was obtained by photolysis of the caged proton 2-NBA (25, 26 ). The photoexcitation of an aqueous solution of 2-NBA with a $355 \mathrm{~nm}$ nanosecond laser pulse leads to the release of protons in high yield $\left(\Phi_{\mathrm{H}}{ }^{+}=0.45\right)$ within a few nanoseconds $(25,27-29)$. The concentration of photoreleased protons can reach the range of $10^{-4} \mathrm{M}$ with laser pulses of a few tens of $\mathrm{mJ}(30,31)$. At low laser pulse energies, the concentration of photoreleased protons is proportional to the laser pulse energy. At each laser pulse energy, the concentration of photoreleased protons can be calculated from the protonation rate (determined through the absorbance changes) of a suitable $\mathrm{pH}$ indicator $(26,32)$. The calibration of the setup and the estimate of the buffering exerted by the protein are described elsewhere (33). This calibration allows the determination of the post-photolysis $\mathrm{pH}$ of the solution, after equilibrium is reached with all reacting species. Under the experimental conditions of this study, the new equilibrium is reached within $1 \mu \mathrm{s}$ after photolysis (33). The single-shot, laser flash photolysis setup for transient absorption was described previously $(30,34)$. The fluorescence emission from NR was excited at $514 \mathrm{~nm}$ with a cw $\mathrm{Ar}^{+}$laser (Uniphase), which served also as the observation light source for the transient absorption measurements. The fluorescence emission was collected from the bottom of the quartz cuvette with a compact photomultiplier (Hamamatsu, H6780), in front of which suitable filters were positioned. In particular, a dichroic mirror with a high reflectivity at $355 \mathrm{~nm}(>99 \%, \mathrm{CVI})$ was employed to remove the stray light from the pump laser used for initiating the $\mathrm{pH}$ jump. A long-pass filter (LOT-Oriel, transmission $90 \%$ at $\lambda>550 \mathrm{~nm}$ ) allowed the selection of fluorescence emission. The current output of the photomultiplier was converted to voltage and amplified by a home-built, DCcoupled broadband amplifier (100 MHz). The voltage signals from the APD and the photomultiplier were fed into the two input channels of a digital sampling oscilloscope (LeCroy 9370, $1 \mathrm{GHz}, 1 \mathrm{Gs} / \mathrm{s})$. To avoid bleaching, the samples were exposed to the intense $514 \mathrm{~nm}$ light only during the time 
necessary for data acquisition using a fast mechanical shutter (Uniblitz). The temperature was controlled by a peltier element with a feedback control mounted below the cuvette holder. This allowed a temperature stability of better than $0.1{ }^{\circ} \mathrm{C}$ to be achieved in the temperature range $10-50{ }^{\circ} \mathrm{C}$. To allow for collection of the fluorescence emission from the bottom of the cuvette, a peltier element with a center hole was employed. The absorbance of 2-NBA at $355 \mathrm{~nm}$ was kept at 2. The concentration of NR was $3 \mu \mathrm{M}$ and the concentration of ApoMb was $30 \mu \mathrm{M}$.

To retrieve kinetic information, fluorescence emission $(F)$ was converted to relative change with respect to the prepulse value $\left(F_{0}\right)$ by taking the ratio

$$
\frac{\Delta F}{F_{0}}=\frac{F-F_{0}}{F_{0}}
$$

Kinetic traces of $\Delta A$ or $\Delta F / F_{0}$ were fitted with the sum of two exponential decay functions using Origin 7.0 (OriginLab).

$\mathrm{p} K_{\mathrm{a}}$ Calculation and Molecular Dynamics Simulations. The crystal structure of sperm whale apomyoglobin complexed with biliverdin $(35,36)$ was obtained from the Brookhaven Protein Data Bank (id code 1bla), with a resolution of $1.5 \AA$. This structure is identical to the holoform of myoglobin because of the presence of the ligand, which prevents the structure from undergoing the structural changes characteristic of the apo-form found in the solution. The structure was used both for the $\mathrm{p} K_{\mathrm{a}}$ calculation and for the MD and docking simulations, after removing the biliverdin and all of the heteroatoms. To model the state of apomyoglobin at $\mathrm{pH} 7$ and 4, we ran different MD simulations under these conditions.

The structure of the intermediate state $I$ is strongly affected by the ionization state of the His, Asp, and Glu residues. Although the $\mathrm{p} K_{\mathrm{a}}$ of several His residues were determined experimentally for the holoprotein, (37) nothing is known about the $\mathrm{p} K_{\mathrm{a}}$ of the carboxylic acids of Glu and Asp residues. We have, therefore, estimated the $\mathrm{p} K_{\mathrm{a}}$ of these titratable groups by means of the program MM_SCP (38, 39). To reach convergence, the damping factor was decreased to 0.15 , and the maximum number of iterations was increased to 1000 . The $\mathrm{pH}$ range of the titration was selected from 2 to 8 , and all hydrogens were considered. Considering an error for the estimated $\mathrm{p} K_{\mathrm{a}}$ of about $0.5,(39)$ the results of the calculation showed that six acidic residues had a $\mathrm{p} K_{\mathrm{a}}$ value above 4 , namely, glutamic acids $41,85,105,109,136$, and 148, (the calculated $\mathrm{p} K_{\mathrm{a}}$ values are $4.6,4.9,4.6,4.7,4.5$, and 4.5 , respectively) whereas the other four carboxylic residues possessed $\mathrm{p} K_{\mathrm{a}}$ values near 4 (Glu38, Glu52, Glu59, and Asp126 for which the calculated $\mathrm{p} K_{\mathrm{a}}$ values are 3.9, 3.9, 3.9 , and 3.8, respectively). Therefore, their protonation state at $\mathrm{pH} 4$ was difficult to define. All of the other acidic residues in the molecule have a $\mathrm{p} K_{\mathrm{a}}$ under 3.5. For these reasons, the structure of ApoMb at $\mathrm{pH} 4$ was determined from two different MD simulations. In the first, only residues 41,85 , $105,109,136$, and 148 were protonated (MD4-6), whereas in the second, all carboxylic acids were protonated for a total of 10 acids in a protonated state (MD4-10). The carboxylate groups were protonated by means of the Sybyl software (Tripos Inc., St. Louis, MO).
The protonation state of His residues was estimated using the experimental $\mathrm{p} K_{\mathrm{a}}$ values available from the literature (37). All His residues were protonated at $\mathrm{pH} 4$ and unprotonated at $\mathrm{pH} 7$, the only exception being His 36 characterized by a $\mathrm{p} K_{\mathrm{a}}$ value around 8 , which was assumed to be in its protonated state at neutral $\mathrm{pH}$.

The MD4 simulations were carried out at higher temperatures to accelerate the unfolding process (40). The first 1000 ps trajectories were run at $300 \mathrm{~K}$, then the temperature was raised to $330 \mathrm{~K}$ between 1000 and $1200 \mathrm{ps}$, and finally, the temperature was set to $360 \mathrm{~K}$ for the remaining time. In all simulations, a stable structure was reached, which showed no appreciable changes during, at least, the last 500 ps. The MD4-6 simulation was run for $1.8 \mathrm{~ns}$, whereas the MD410 simulation was run for $2.5 \mathrm{~ns}$.

The trajectory at neutral $\mathrm{pH}(\mathrm{MD} 7)$ was run under the same temperature conditions and for the same time to investigate the structural changes in apomyoglobin free in solution and be able to distinguish the effect of $\mathrm{pH}$ on the protein structure and dynamics from the structural relaxation of the apo-form. The structure became stable before the increase of the temperature and subsequently did not change significantly. Accordingly, the mean structure was calculated over the last $300 \mathrm{ps}$ before the temperature was increased to $330 \mathrm{~K}$.

The starting structures for the MD simulations were first minimized with the quasi-Newton algorithm until an energy gradient value of 0.1 and afterward with a truncated Newton method until a 0.01 gradient value. The energy minimizations and the MD simulations were performed with the Tinker package (41), using the Charmm force field (42) and adding the parameters of the protonated groups from the Charmm software package. The importance of correctly accounting for solvation effects during MD simulations has been widely discussed. Because explicit treatment of the solvent is time consuming, and perhaps not necessary, several implicit methods have been proposed to account for solvation effects in proteins $(43,44)$. We used the default settings of the Tinker software, which keeps a constant $\epsilon=1$ and uses a macroscopic solvation potential energy term (the GB/SA solvation model was selected (45)). A cut off radius for nonbonding interactions was switched from 8 to $10 \AA$. The bond length was kept fixed for all atoms. A time step of 2 fs was employed.

For all the simulations a mathematical mean structure was calculated using the structures saved in the last ps, where the structure was stable, by means of the MolMol software (46). A root-mean-square deviation (rmsd) was evaluated between this structure and all of the structures used for its calculation. The structure with the lowest rmsd value was chosen as a mean structure and used for all further structural analyses. Herein, we will refer to this structure as the mean structure. The helicity and the secondary structure content were estimated with the DSSP algorithm (47). The solventaccessible surface area, SASA, (using a probe sphere of 1.4 $\AA$ radius), the total, and per residue backbone rmsd's compared with those of the crystal structure were calculated using the MolMol software. Both the global and local contributions of the backbone rmsd per residue were calculated. The global rmsd per residue gives the displacement of each residue from the reference structure, after superimposing the whole structure. The local rmsd per residue gives the displacement of each residue from the 
reference structure, after superimposing three residues centered at the considered residue. The comparison of the global and local contribution to the rmsd per residue value is useful to discriminate between a spatial shift of a structured region and a local unfolding.

The convergence of the simulations was checked by plotting, as a function of time, the potential energy, the local and global rmsd's, the backbone rmsd of the total structure, the helicity, and the SASA. All of these parameters were unchanged over the last 500 ps of the simulations and demonstrated the convergence of the simulations. Details can be found in the Supporting Information.

The identification and characterization of the size of the pockets, cavities, and mouth openings were made by means of the CASTp server $(48,49)$ (http://cast.engr.uic.edu/cast). The figures were generated with the VMD program (50) and with the ViewerLite 5.0 (Accelrys Software, Inc.) software.

Docking Simulations. Simulations of the interaction of the NR molecule with ApoMb in the different conformations were performed with the Autodock3 software package (51) and the AutoDockTools (ADT) interface. The NR molecule was built with the sketch routine of the Sybyl software and refined with minimization.

The partial atomic charges for the ligands were added according to Gasteiger and Marsili (52). All rotatable bonds were kept free to rotate. The Kollman united-atom partial atomic charges (53) were added to the ApoMb molecules, taking into account the protonation state of each residue. For the docking calculation, the Lamarckian genetic algorithm was used, performing 100 runs with 150 individuals in a population, 27 generations, and 1000000 energy evaluations. The maximum translation step was set to $2 \AA$, the maximum quaternion step to $50^{\circ}$, and the maximum torsion step to $50^{\circ}$.

The grid maps were centered on the center of mass of the macromolecule, with a spacing of $0.375 \AA$ and a number of points in the $x, y$, and $z$ directions of 106, 114, 96, respectively.

\section{RESULTS}

Steady-State Fluorescence Emission. NR in water is characterized by a low fluorescence emission $\left(\Phi_{\mathrm{F}} \approx 0.02\right.$ (20)) centered at $665 \mathrm{~nm}$. When apomyoglobin is added to a neutral, aqueous ( $\mathrm{pH} 7$ ) solution of NR, the fluorescence emission intensity shows a substantial increase and a remarkable blue shift with a maximum located at $628 \mathrm{~nm}$. When the $\mathrm{pH}$ is lowered to 4 , at constant protein concentration, the fluorescence emission further blue shifts to $610 \mathrm{~nm}$ while the intensity decreases.

Figure 1 compares the fluorescence emission spectra of aqueous solutions of NR $(300 \mathrm{nM})$ in water and in the presence of apomyoglobin at $\mathrm{pH} 4(93 \mu \mathrm{M})$ and $7(85 \mu \mathrm{M})$. A blue shift and an increase in fluorescence emission with respect to water have been used in the past as evidence of the interaction of the dye with the protein (19).

The observed fluorescence enhancement and spectral blue shift of the emission of NR when apomyoglobin is present in solution unequivocally shows that the dye binds to a hydrophobic pocket of the protein.

The natural candidate as the binding site for the dye on the apoprotein is the heme pocket, which, in the apoprotein, is empty and relatively solvent exposed. Figure 2 compares

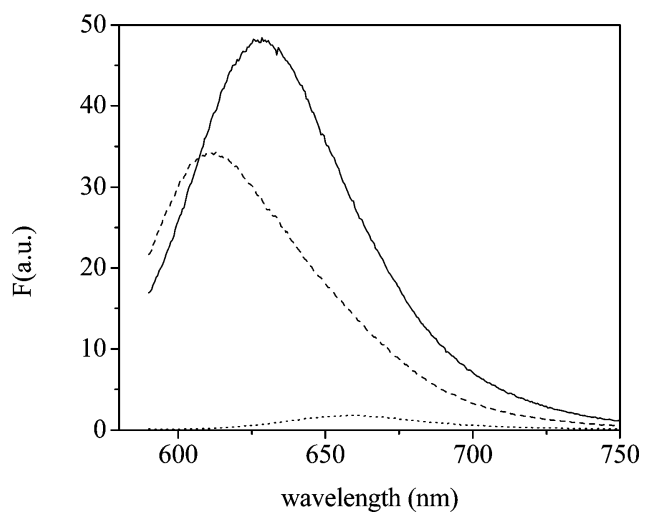

FIGURE 1: Fluorescence emission of aqueous solutions of NR (300 $\mathrm{nM})$ alone (....) and in the presence of ApoMb at $\mathrm{pH} 7(85 \mu \mathrm{M})$ $(-)$ and $\mathrm{pH} 4(93 \mu \mathrm{M})(---)$ at $T=20^{\circ} \mathrm{C}$. The excitation wavelength was $580 \mathrm{~nm}$.

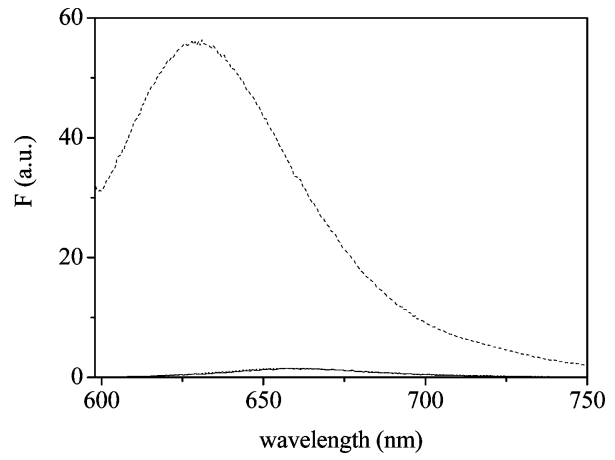

FIGURE 2: Fluorescence emission of a solution of NR $(0.2 \mu \mathrm{M})$ alone (....), in the presence of ApoMb $(30 \mu \mathrm{M})(---)$, and in the presence of myoglobin $(30 \mu \mathrm{M})(-)$ at $\mathrm{pH} 7$ and $T=20^{\circ} \mathrm{C}$. The excitation wavelength was $580 \mathrm{~nm}$.

the fluorescence emission spectra of neutral aqueous solutions of $\mathrm{NR}$ in the presence of ApoMb and myoglobin at equal protein concentrations. The fluorescence emission of the dye in the presence of myoglobin is indistinguishable from the emission in water.

The absence of effects on the fluorescence emission of the dye when myoglobin is added is a strong suggestion that the binding site is not present in the holoprotein. These experimental findings suggest the idea that the binding site is located at the heme pocket. The results from the molecular docking studies (vide infra) give strong support to this hypothesis, with a very defined and specific affinity of NR for the heme pocket.

It has been previously proposed that other hydrophobic dyes bind to the heme pocket of apomyoglobin. ANS has been used to monitor structural fluctuations attributed to the heme pocket $(3,54)$. Fluorescence correlation spectroscopy has revealed ANS dynamics, which have been attributed to the binding of the dye to the hydrophobic heme pocket, both in horse heart and tuna myoglobin. The observed dynamics were attributed to the binding of the dye to the protein because it was found to be independent of the concentrations of the reactants. The observed rates were found to be governed by the dissociation rates. At low $\mathrm{pH}$, an additional binding site appeared to be available for the dye. Pyranine has been used to study proton-transfer dynamics within the heme pocket of apomyoglobin (55-57). However, as detailed in the next section, this is the first report showing support from numerical simulations to the hypothesis, on the basis 


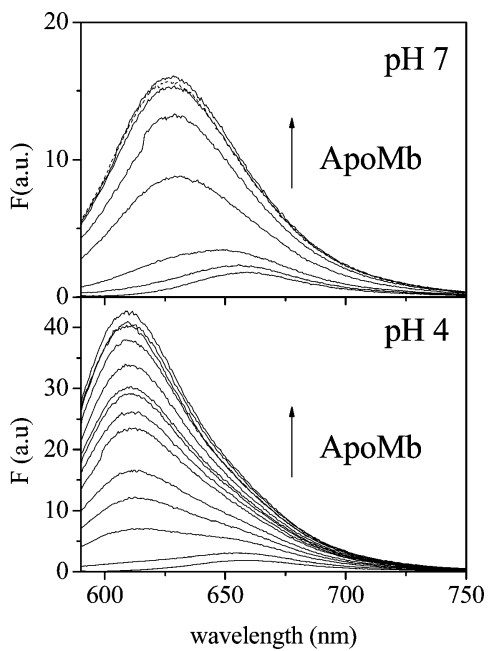

FIGURE 3: Fluorescence emission of NR $(0.2 \mu \mathrm{M})$ as a function of ApoMb concentration at $\mathrm{pH} 7$ and 4 . The concentration of ApoMb was increased from 0 to $82 \mu \mathrm{M}$ at $\mathrm{pH} 7$ and from 0 to $320 \mu \mathrm{M}$ at $\mathrm{pH} 4$ and $T=20^{\circ} \mathrm{C}$. The excitation wavelength was $580 \mathrm{~nm}$.

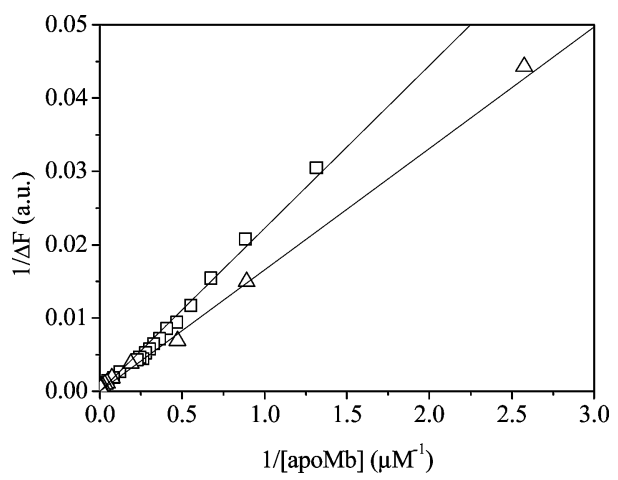

FIGURE 4: Double reciprocal plot of the observed changes in fluorescence emission as a function of protein concentration at $\mathrm{pH}$ $4(\triangle)$ and $7(\square)$. The fluorescence emission at each concentration was estimated from the whole area under the corresponding spectrum in Figure 3.

of the experimental data, as to the identification of the heme pocket as the binding site for the dye.

To determine the affinity of the dye for the apoprotein in the native and in the molten globule states, we have measured the fluorescence emission of the dye at increasing protein concentrations at $\mathrm{pH} 7$ and 4 . Figure 3 compares the fluorescence emissions of NR in the presence of apomyoglobin at $\mathrm{pH} 4$ and 7 at various protein concentrations. In both cases, the fluorescence emission increases and the emission maximum blue-shifts as the protein concentration is increased. At high protein concentrations, the fluorescence intensities of both samples reach saturating values and show emission bands centered at $628 \mathrm{~nm}$ at $\mathrm{pH} 7$ and $610 \mathrm{~nm}$ at $\mathrm{pH} 4$. Only two spectrally distinct species were detectable for each titration by singular-value-decomposition analysis (58) (Supporting Information). One species can be identified as NR free in solution and the other as NR bound to a hydrophobic environment, which is different at $\mathrm{pH} 4$ and 7.

From these data, we have estimated the dissociation constants for the $\mathrm{NR}-\mathrm{ApoMb}$ complexes in the native state $(\mathrm{pH} 7)$ and in the molten globule state $(\mathrm{pH} 4)$ of the protein, by plotting (Figure 4), in a double reciprocal plot, the fluorescence emission change and the protein concentration (24). The resulting dissociation constants are $25 \pm 5 \mu \mathrm{M}$ at

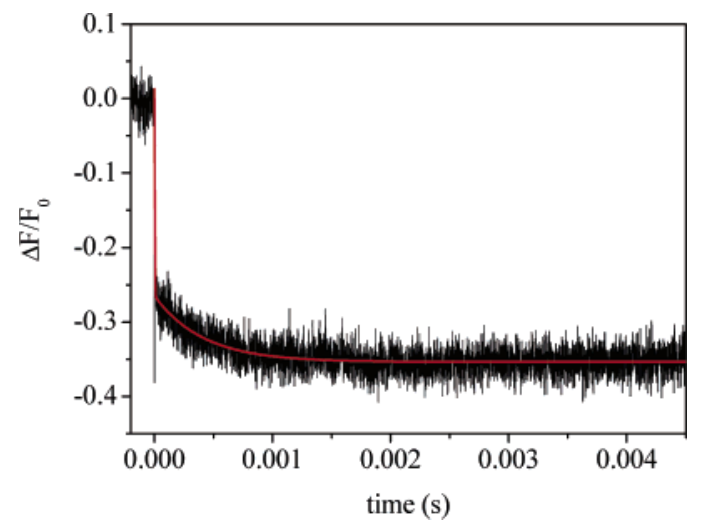

FIGURE 5: Transient fluorescence emission intensity for a solution containing 2-NBA, NR $(3 \mu \mathrm{M})$, and ApoMb $(30 \mu \mathrm{M})$ at pre-pulse $\mathrm{pH} 7$ following a single, $71 \mathrm{~mJ}$ laser pulse at $355 \mathrm{~nm} ; T=20^{\circ} \mathrm{C}$. The red curve is the result of the fit with a double exponential decay.

$\mathrm{pH} 7$, and $52 \pm 5 \mu \mathrm{M}$ at $\mathrm{pH} 4$. An alternative estimate of the dissociation constants can be found in the Supporting Information and gives a consistent 2-fold increase in the $K_{\mathrm{d}}$ value upon lowering the $\mathrm{pH}$ from 7 to 4 .

From the data extrapolated at very high protein concentrations, where virtually all of the dye molecules are bound to protein molecules, it is possible to estimate the fluorescence quantum yields of NR bound to the native state and the molten globule state using the quantum yield in $\mathrm{H}_{2} \mathrm{O}\left(\Phi_{\mathrm{F}}=\right.$ 0.02) (20). The quantum yield of NR bound to ApoMb at $\mathrm{pH} 7$ is $0.22 \pm 0.02$ and shows a 10 -fold enhancement with respect to an aqueous solution. It further increases to 0.52 \pm 0.08 at $\mathrm{pH} 4$, indicating that in the molten globule state a more hydrophobic pocket becomes available. This finding is consistent with the docking results, which are reported in the sections that follow. The increased fluorescence quantum yield at $\mathrm{pH} 4$ is in agreement with the spectral shift observed under the same conditions (Figure 1). The apparent decrease in the fluorescence emission intensity at constant protein concentrations is due to the lower affinity of the dye for the protein at $\mathrm{pH} 4$ (vide supra).

Laser pH-Jump. To estimate the rate constant for the dissociation of the $\mathrm{NR}-\mathrm{ApoMb}$ complex when the $\mathrm{pH}$ is lowered below neutrality, we have performed laser $\mathrm{pH}$-jump experiments $(26,30)$. Figure 5 shows the time course of the fluorescence emission intensity of NR in the presence of apomyoglobin after a single, $71 \mathrm{~mJ}$ laser flash at $355 \mathrm{~nm}$ has been impinged onto the sample. The fluorescence emission intensity decreases after the laser flash by approximately $35 \%$.

The time-resolved signal at $20{ }^{\circ} \mathrm{C}$ can be well described by a double exponential decay with lifetimes of $2.7 \pm 0.2$ $\mu \mathrm{s}$ and $350 \pm 5 \mu \mathrm{s}$, at all laser pulse energies employed in this study. Thus, the lifetimes are not influenced by the extent of the $\mathrm{pH}$ jump, suggesting that we are observing reactions with rates that are not limited by the protonation of the protein. This shows that NR is monitoring $\mathrm{pH}$-induced changes of the protein, which occur on the microsecond time scale. Although the amplitude of the slow component is scarcely affected by the $\mathrm{pH}$ reached after the laser flash, the amplitude of the fast phase is very sensitive to this parameter. This is evident from the data in Figure 6, where the amplitudes of the two exponential decays are plotted as a 


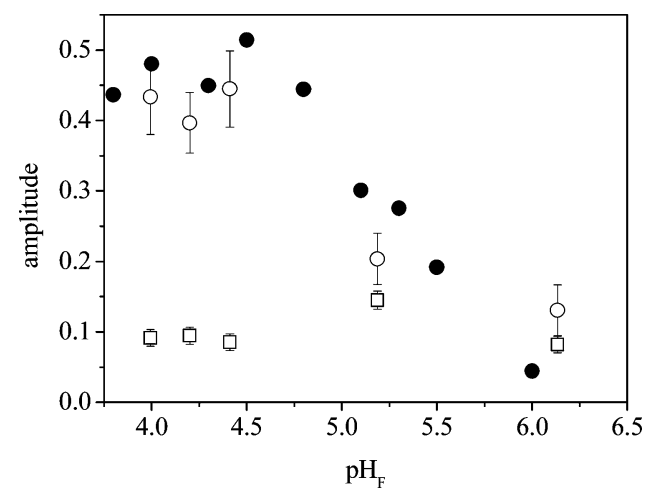

FIGURE 6: Amplitudes of the exponential decays $(\bigcirc 2.7 \pm 0.2 \mu \mathrm{s}$, $\square 350 \pm 5 \mu \mathrm{s}$ ) obtained from the fit of the changes in fluorescence emission, following a single laser flash as a function of the final $\mathrm{pH}$ at $20^{\circ} \mathrm{C}$. The final $\mathrm{pH}$ decreases as the laser pulse energies are increased. The laser pulse energies used in this experiment were $10,21,49,71$, and $114 \mathrm{~mJ}$. The fractional decrease of fluorescence with respect to the emission observed at $\mathrm{pH} 7$ in a steady-state experiment is shown as $(\bullet)([\mathrm{NR}]=0.2 \mu \mathrm{M}$, [ApoMb] $=68 \mu \mathrm{M})$.

function of the postphotolysis $\mathrm{pH}$. A comparison of the two decays with the observed steady state change in fluorescence shows that the change observed in our experiments accounts for the whole change at equilibrium. Thus, the structural changes sensed by NR are complete within a few milliseconds. The few pH-jump experiments performed on $\mathrm{ApoMb}$ with submillisecond resolution monitored only volume changes accompanying the unfolding reaction $N \rightarrow$ $I$, suggesting that most of the large volume change accompanying the formation of the $I$ state (12) is produced within a few microseconds (13), with very little, if any, additional volume changes on the millisecond time scale (14). The $\mathrm{pH}$ dependence of the amplitude of the fast component (Figure 6) and its lifetime $(2.7 \mu \mathrm{s})$ both suggest that this transient directly senses the early events of the $N \rightarrow I$ transition, whereas the slower component may reflect a subsequent rearrangement of NR, possibly, to the final location inside the new conformation of the protein. The structural changes at the source of the kinetics reported by $\mathrm{NR}$ are not easily assigned at present, and the assignment of the observed kinetics to specific molecular events is difficult. The temperature dependence of the observed rate constants allows the drawing of linear Arrhenius plots from which we estimate, for the fast transient, an enthalpic barier of $4 \pm 1 \mathrm{kcal} / \mathrm{mol}$, whereas the slower decay has a much higher barrier of $15.9 \pm 0.3 \mathrm{kcal} / \mathrm{mol}$.

Molecular Dynamics Simulations. MD simulations at neutral and acidic $\mathrm{pH}$ were performed mainly to determine the atomic coordinates of intermediate $I$ at $\mathrm{pH} 4$ and $\mathrm{ApoMb}$ at neutral $\mathrm{pH}(N)$. This structural information is essential for the characterization of the interaction with NR. In addition, the modeling of the protonation state of apomyoglobin at $\mathrm{pH} 4$ revealed the important contributions from specific residues to the structural changes leading to the wellknown unfolding intermediate $I$.

Two unfolding simulations at $\mathrm{pH} 4$, with 6 and 10 acidic residues in the neutral form (MD4-6 and MD4-10, see Materials and methods) were carried out. At $\mathrm{pH} 7$, the net charge is +3 . As expected on the basis of the smaller charge on the molecule, the mean structure reached with the MD4-6 is slightly less distorted compared to the one with MD410. In particular, even if the helical content, measured as
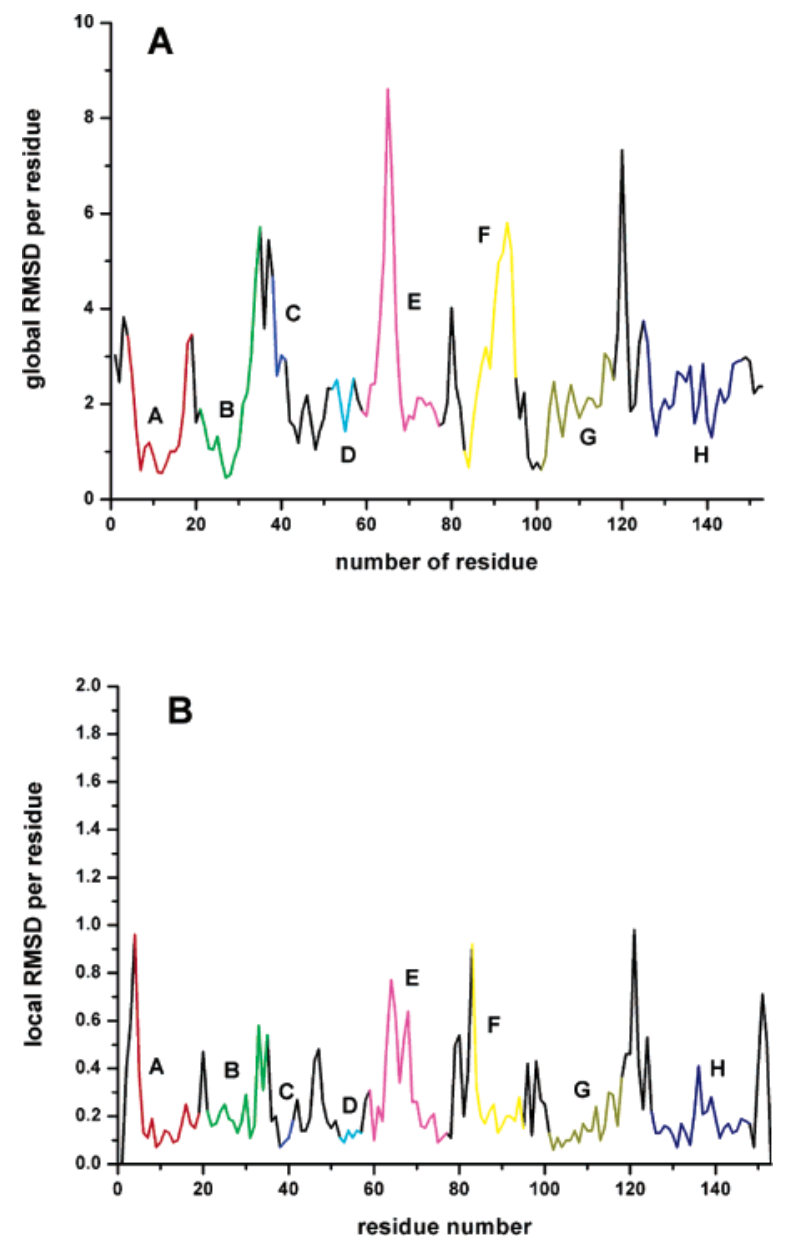

FIGURE 7: Global (A) and local (B) backbone rmsd per residue between the MD4-10 mean structure and the crystal. The eight helices, assigned with the DSSP method to the crystal structure, are labeled and indicated with different colors.

the number of $\alpha$-helix hydrogen bonds, is the same, the collapse of the binding pocket is not as extended. This is evident from the measure of the volume of the binding pocket for the MD4-6 structure, which is smaller than the one observed for the MD4-10 structure ( 893 vs $1359 \AA^{3}$ ). The analysis of the relative helicity and the structural characteristics of the mean structure obtained with MD4-10 were compatible with the characteristics expected for the intermediate $I$; therefore, we analyzed only this last structure.

The relative helicity of the MD4-10 mean structure, that is, the $\alpha$-helical content relative to that of the $\mathrm{Mb}$ crystal structure, is in very good agreement with general experimental observations. $(9,11,59-61)$ Assuming that the helicity of the holoprotein $(\mathrm{Mb})$ is 1 the MD4-10 mean structure has a calculated helicity of 0.58 , whereas the experimental results give a value of 0.54. (9) From the local and global backbone rmsd per residue (Supporting Information, Figure S5) values and the structural superposition of the MD4-10 mean structure with the crystal structure (Figures 7 and 8A), we can say that the helices forming the heme binding pocket (E, F, and $\mathrm{C}$ and partially helix B) collapse into the free cavity of the binding site. Figure 7 also shows that helix $\mathrm{C}$ only shifts, whereas helices $\mathrm{E}$ and $\mathrm{F}$ and the $C$-terminus of helix B are only partly unfolded. Helices A, D, G, and $\mathrm{H}$ maintain a significant amount of helical structure (61). These results agree well with recent evaluations of the helical content of state $I$, which seem to 


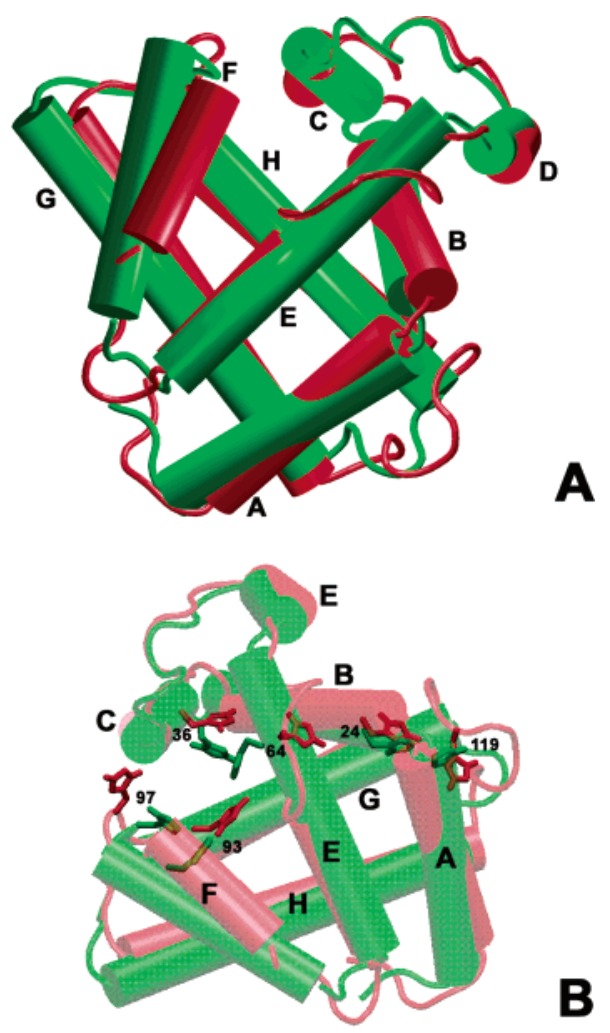

FIGURE 8: (A) Cartoon rendering of the structures from the crystal (green) and MD4-10 (red) with the helices labeled and represented as cylinders. (B) the cylinders are drawn in a semitransparent rendering to highlight the His residues represented as sticks.

override the previous consensus based mainly on hydrogendeuterium exchange experiments on the static intermediate at $\mathrm{pH} 4.2$ (1). Heteronuclear NMR investigations demonstrated that besides $\mathrm{A}, \mathrm{G}$, and $\mathrm{H}$ helices $\mathrm{B}, \mathrm{C}, \mathrm{D}$, and $\mathrm{E}$ also show a partial helical conformation at $\mathrm{pH} 4.2(59,61)$. Recent continuos flow, time-resolved circular dichroism, and SAXS experiments confirmed that the observed rapid $(<300 \mu \mathrm{s})$ collapse of the unfolded protein into state $I$ cannot be explained only by the formation of the A, G, and $\mathrm{H}$ helices, suggesting the involvement of additional regions of the protein (62). Finally, quench-flow hydrogen exchange experiments showed a pattern of protected amide protons, which is consistent with the structural heterogeneity in the kinetic molten globule, with helices showing refolding on different time scales. The sites include, besides the E helix, the ends of the A and B helices and part of the C helix (63).

His residues deserve particular consideration. In the crystal structure, all His residues are rather exposed to the solvent, except for those involved in important interactions stabilizing the protein structure. The latter include His 93 (the distal His), His64 (the proximal His), His24, His119 (the two His forming the $\mathrm{H}$ bond important for the integrity of the $\mathrm{N}$ form), and, partially, His36 and His97, which are placed at the edge of the binding pocket (Figure 8B). During acidic unfolding, because of the electrostatic repulsive force, His residues of the two pairs, His93/His64 and His24/His119, move away from each other. His 93 and His64 are located within the unfolded regions of the two $\alpha$-helices they belong to ( $F$ and $\mathrm{E}$, respectively). His 93 becomes much more solvent exposed than in the crystal. His97 lays in a loop connecting helices $\mathrm{F}$ and $\mathrm{G}$, which becomes more flexible upon unfolding and could, perhaps, regulate access to the binding site. The $\mathrm{H}$ bond between His 24 and His119 is broken, and the two residues separate, unfolding the extremities of the two helices, B and G, where they are located. On the contrary, in the MD7 structure, the positions of His64, His93, His24, and His119 do not vary significantly, with the $\mathrm{H}$ bond between His24 and His119 being unperturbed. In the structure at $\mathrm{pH} \mathrm{4}$, all histidine residues are situated in unfolded regions. This fact confirms that because of their net charge histidine residues play a major role in the first step of ApoMb unfolding leading to the $\mathrm{pH} 4$ intermediate.

Results from MD7 show that at neutral $\mathrm{pH}$ ApoMb adopts a structure only slightly distorted with respect to that of the crystal, the only remarkable difference being in the collapse of the helices into the binding pocket. This fact is confirmed by the global rmsd plot per residues (Supporting Information, Figure S3), showing that the collapse occurs mainly in the region of the $\mathrm{C}$ and $\mathrm{D}$ helices. The corresponding local rmsd plot per residue (Supporting Information, Figure S3) indicates that the variation is due to a spatial movement more than to a local unfolding, which mainly concerns the connection loops between helices. The relative helicity for this structure is 0.75 , which is in very good agreement with the experimental value of 0.77 (9). The variation with time of the backbone rmsd of the total structure and solvent-accessible surface (SASA) (Supporting Information, Figure S4) shows the convergence of the simulation.

Docking Simulations. The interaction of the probe NR with ApoMb was investigated by means of molecular docking simulations for the structures available from the crystal, MD4-10 and the MD7 (vide supra). The best conformations obtained, scored on the basis of the docked energy (51), are all statistically well represented.

The docking with the crystal structure shows that the ligand finds its best conformation into the binding site of the heme group, with an orientation bringing the rings of the probe to overlap the place where two of the hemeporphyrin rings would be if the heme were present. The environment of the ligand shows both hydrophobic and polar features, and one $\mathrm{H}$ bond is formed between the $\mathrm{N}$ atom of the diethylamino group and the backbone $\mathrm{O}$ atom of the protein residue Lys42 (Figure 9). The residues belonging to the walls of the binding pocket, which are present within a $7.0 \AA$ radius around NR, are listed in Table 1. Among the hydrophobic residues, a key role is played by Phe43, which is stacked with the last ring of NR, and Leu104 and Ile107, which surround the more hydrophobic NR ring. The relevant polar residues surrounding NR, in addition to Lys 42 , are the triad Asp44, Arg45, and Lys96, surrounding the NR tail; the distal His93 and the proximal His64; His97, and the more distant His36. Ten of the 17 residues surrounding NR also belong to the mouth of the binding pocket.

The docking of NR with the ApoMb structure at $\mathrm{pH} 4$ shows that the dye binds to the heme pocket (Figure 10). The resulting dissociation constants were $K_{\mathrm{d}}=1.5 \mu \mathrm{M}$ for the crystal structure, and $K_{\mathrm{d}}=2.6 \mu \mathrm{M}$ for the MD4-10 structure, suggesting a higher affinity of NR for the crystal structure, as shown by the fluorescence emission data. Although the computed $K_{\mathrm{d}}$ values are on the same order of magnitude as the experimental ones, the error associated with the numerical estimate prevents any quantitative comparisons $(51,64)$. 


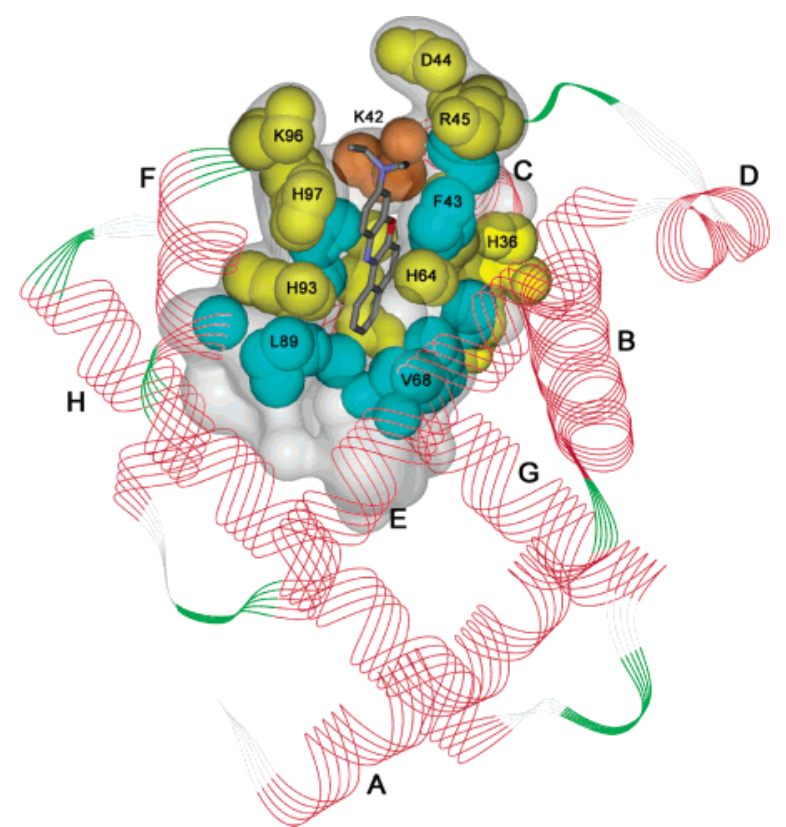

FIGURE 9: Neighborhood of NR in the crystal complex (in a line ribbons representation and colored by secondary structure). The polar residues (in the van der Waals radius representation), yellow; hydrophobic residues, cyan; and the Lys 42 residue, orange. The helices and relevant residues are labeled. The whole binding-pocket Connolly's surface is in light gray and in semitransparent rendering.

Table 1: Residues that Are Present within a 7.0 ̊ Radius around NR in the Different Complexes

\begin{tabular}{cccc}
\hline & crystal-NR & $\begin{array}{c}\text { MD4-10 } \\
\text { structure-NR }\end{array}$ & $\begin{array}{c}\text { MD7 } \\
\text { structure-NR }\end{array}$ \\
\hline \multirow{4}{*}{ Leu32 } & Phe33 & Phe33 \\
& Phe43 & Phe43 & Phe43 \\
& Ile99 & Phe46 & Phe46 \\
& Leu104 & Val68 & Val68 \\
& Ile107 & Ile107 & Pro88 \\
hydrophobic & Val68 & Leu29 & Leu89 \\
& Leu89 & Leu32 & \\
& & Leu61 & \\
& & Leu69 & \\
& & Leu72 & \\
& & Leu89 & \\
& Lys42 & Asp60 & Asp44 \\
Asp44 & Arg45 & Arg45 \\
Arg45 & Lys63 & Asp60 \\
& Thr39 & His93 & Lys63 \\
& Lys96 & His64 & His64 \\
& Tyr103 & Thr67 & Thr67 \\
& His64 & Tyr103 & Ser92 \\
& His93 & Thr70 & His93 \\
& His97 & & Lys96 \\
His36 & & & His97
\end{tabular}

${ }^{a}$ The distance for this residue was $8 \AA$.

The NR bound to ApoMb at $\mathrm{pH} 4$ is positioned in a different orientation, reaching a more hydrophobic environment. This finding is consistent with the fluorescence emission spectrum, which, under these conditions, shows a blue shifted peak at $610 \mathrm{~nm}$ (Figure 1). No $\mathrm{H}$ bonds are formed with the protein in agreement with the higher binding energy and $K_{\mathrm{d}}$. The neighborhood of the ligand shows a cluster of hydrophobic residues into which NR penetrates. The amino acids that constitute this hydrophobic cluster are listed in Table 1. In particular, there are three phenylalanine residues (Phe33, Phe43, and Phe46), pointing toward NR

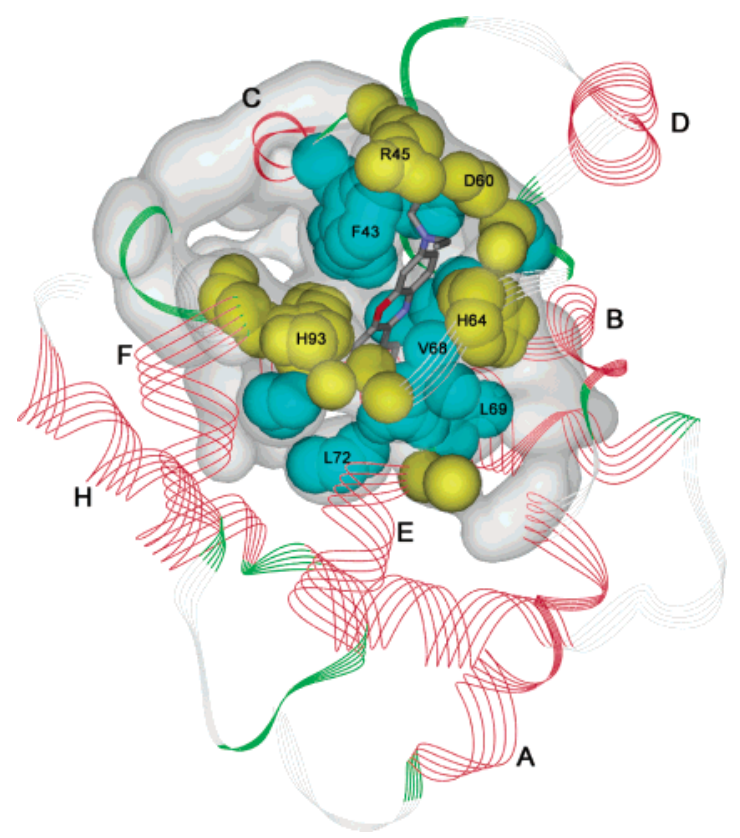

FIGURE 10: Neighborhood of NR in the MD4-10 structure complex (in a line ribbons representation and colored by secondary structure). The polar residues (in the van der Waals radius representation) are in yellow and hydrophobic ones are in cyan. The helices and relevant residues are labeled. The whole bindingpocket Connolly's surface is in light gray and in semitransparent rendering.

from the same side, and several Leu residues $(29,32,61$, 69,72 , and 89) surrounding the dye. The polar residues present within a $7.0 \AA$ radius around NR are also listed in Table 1. Asp60, Arg45, and Lys63 surround NR with a similar arrangement to the one observed for Asp44, Arg45, and Lys96 in the crystal structure; the proximal His64 and the distal His93, which with Thr67 is placed close the O-atom of the NR central ring, are also present. Only some residues in the neighborhood of NR are the same as those observed for the crystal, but in general, the polarity of the residue is preserved. From an inspection of the MD4-10 mean structure (Figures 8B and 10) it seems that the repulsive forces between the charged His residues 64, 93, and 97, located at the edge of the binding pocket, make the cavity accessible, although more tight, thus allowing the ligand to enter more deeply into the binding site. In fact, only five of the nineteen residues surrounding NR belong to the mouth of the binding pocket. If we compare the calculated volumes of the binding pockets of the crystal structure and the MD410 structure (Table 2), we can see that the latter is larger. On the contrary, the area of the mouth opening is smaller than that in the crystal pocket. It seems that in the MD4-10 structure, although on the one hand the binding pocket becomes larger because of the helices unfolding, on the other hand, the collapse of the helices narrows the outer region of the pocket.

Although the docking simulation of NR with the crystal structure does not find any conformation outside the heme binding pocket, in the MD4-10 structure (and also in the docking with the MD7 structure), a second, less favorable site is found on the surface of the G helix. This could be due to the deformation of the binding pocket with the collapse of the helices that make the position on the G helix 
Table 2: Areas and Volumes of the Binding Pockets and the Areas of Their Mouths for the Three Analyzed Structures as Identified by the CASTp Server ${ }^{a}$

\begin{tabular}{llc}
\hline structure & $\begin{array}{c}\text { volume of } \\
\text { the binding } \\
\text { pocket }\left(\AA^{3}\right)^{b}\end{array}$ & $\begin{array}{c}\text { area of } \\
\text { the mouth } \\
\left(\AA^{2}\right)^{c}\end{array}$ \\
\hline crystal & 970 & 180 \\
MD4-10 & 1359 & 149 \\
MD7 & $226+135^{d}$ & 0 \\
\hline
\end{tabular}

${ }^{a}$ See Material and Methods. ${ }^{b}$ Surrounded by a Connolly's molecular surface. A solvent probe of $1.4 \AA$ was used for the calculation. ${ }^{c}$ Measure of the area of the pore or mouth opening of the pocket. ${ }^{d}$ Volumes of the two adjacent cavities into which the binding pocket is split.

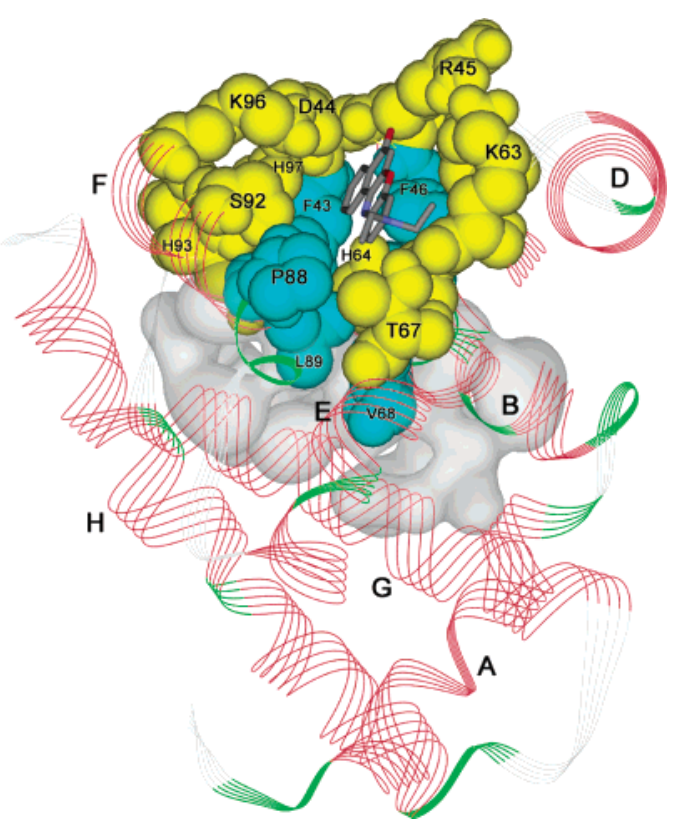

FIGURE 11: Neighborhood of NR in the MD7 structure complex (in a line ribbons representation and colored by secondary structure). Polar residues (in the van der Waals radius representation) are in yellow and the hydrophobic ones are in cyan. The helices and relevant residues are labeled. The whole binding-pocket Connolly's surface is in light gray and in semitransparent rendering.

side more accessible because of the displacement of the $\mathrm{C}$ helix and the $C$-terminus of the B helix.

Unlike the previous docking, the complex with the neutral ApoMb (pH 7) obtained from MD7 is more unfavorable than the NR-crystal complex (Figure 11). The binding energy of the best conformation is higher, and the estimated $K_{\mathrm{d}}$ values show an enhancement of about one order of magnitude. The position of the ligand is much more external and in a different orientation. The NR neighborhood is very polar, with few hydrophobic residues and, therefore, quite unfavorable for such a hydrophobic molecule. From the structure in Figure 11, it is apparent that the collapse of the helices is not accompanied by an increased accessibility of the binding pocket (unlike the case of the acidic complex), which, in fact, splits into two closed cavities that are adjacent but separated that ligands cannot reach.

The reason for this disagreement in the change of $K_{\mathrm{d}}$ between $\mathrm{pH} 4$ and 7 when the MD7 structure is considered may be found in the interaction between the ligand and the protein, which is likely affecting the structure of the protein. It is reasonable to envision that NR may induce a partial refolding of the solution structure of the apoprotein in a way similar to that observed when $\mathrm{Mb}$ is reconstituted from the apoprotein and the heme (23). If this is the case, the docking simulation of NR with the neutral ApoMb structure is meaningless because it is not able to simulate the flexibility of the receptor structure that could trigger the refolding reaction. The refolding of the structure at neutral $\mathrm{pH}$ in the presence of the ligand could be favored by the presence of the $\mathrm{H}$ bond between His 24 and His 119, which is preserved. This $\mathrm{H}$ bond is broken in the MD4-10 structure; therefore, presumably, it is more difficult for the ligand to affect the structure of the apoprotein inducing a partial refold.

\section{CONCLUSIONS}

The computational characterization at the atomic level of the $\mathrm{pH} 4$ intermediate structure of ApoMb highlighted the effects of the protonation of amino acid residues, mainly, histidines, on the binding pocket volume and polarity.

Fluorescence emission experiments and computational docking simulations show that the polarity probe NR binds to ApoMb within the heme binding pocket. The affinity of $\mathrm{NR}$ for the protein is sensitive to the folding of ApoMb, being higher for the native than that for the molten globule state. The location of NR in the molten globule state is in a more hydrophobic pocket. The docking of NR with the crystal structure shows that the ligand binds to the same site of the heme group with an orientation that brings the rings of the probe to overlap two of the heme porphyrin rings. The docking of NR with the ApoMb structure at $\mathrm{pH} 4$ shows that the dye binds to the heme pocket with a slightly less favorable binding energy, in keeping with the lower value of the experimental $K_{\mathrm{d}}$. Under these conditions, the NR is positioned in a different orientation, reaching a more hydrophobic environment.

Besides monitoring the steady-state properties of the ApoMb structure, NR is capable of tracking kinetics in the microsecond range associated with the transition from the native to the molten globule state induced by a nanosecond laser $\mathrm{pH}$ jump.

\section{ACKNOWLEDGMENT}

Centro Interfacoltà Misure of the University of Parma is acknowledged for the use of the circular dichroism facility.

\section{SUPPORTING INFORMATION AVAILABLE}

Extraction of fluorescence spectra for reacting species by singular value decomposition, convergence of MD simulations, and the location of NR inside the binding pocket. This material is available free of charge via the Internet at http://pubs.acs.org.

\section{REFERENCES}

1. Hughson, F. M., Wright, P. E., and Baldwin, R. L. (1990) Structural characterization of a partly folded apomyoglobin intermediate, Science 249, 1544-1548.

2. Barrick, D., and Baldwin, R. L. (1993) The molten globule intermediate of apomyoglobin and the process of protein folding, Protein Sci. 2, 869-876.

3. Cocco, M. J., and Lecomte, J. T. J. (1994) The native state of apomyoglobin described by proton NMR spectroscopy: interaction 
with the paramagnetic probe HyTEMPO and fluorescent dye ANS, Protein Sci. 3, 267-281.

4. Johnson, R. S., and Walsh, K. A. (1994) Mass spectrometric measurement of protein amide hydrogen exchange rates of apoand holo-myoglobin, Protein Sci. 3, 2411-2418.

5. Privalov, P. L. (1996) Intermediate states in protein folding, $J$. Mol. Biol. 258, 707-725.

6. Hughson, F. M., Barrick, D., and Baldwin, R. L. (1991) Probing the stability of a partly folded apomyoglobin intermediate by sitedirected mutagenesis, Biochemistry 30, 4113-4118.

7. Waltho, J. P., Feher, V. A., Merutka, G., Dyson, H. J., and Wright, P. E. (1993) Peptide models of protein folding initiation sites. 1. Secondary structure formation by peptides corresponding to the G-helix and H-helix of myoglobin, Biochemistry 32, 6337-6347.

8. Barrick, D., Hughson, F. M., and Baldwin, R. (1994) Molecular mechanism of acid denaturation of apomyoglobin. The role of hystidine residues in the partial unfolding of apomyoglobin, $J$. Mol. Biol. 237, 588-601.

9. Onufriev, A., Case, D. A., and Bashford, D. (2003) Structural details, pathways, and energetics of unfolding apomyoglobin, $J$. Mol. Biol. 325, 555-567.

10. Jennings, P. A., and Wright, P. E. (1993) Formation of a molten globule intermediate early in the kinetic folding pathway of apomyoglobin, Science 262, 892-896.

11. Eliezer, D., Jennings, P. A., Wright, P. E., Doniach, S., Hodgson, K. O., and Tsuruta, H. (1995) The radius of gyration of an apomyoglobin folding intemediate, Science 270, 487-488.

12. Vidugiris, G. J. A., and Royer, C. A. (1998) Determination of the volume changes for pressure-induced transitions of apomyoglobin between the native, molten globule, and unfolded states, Biophys. J. $75,463-470$.

13. Abbruzzetti, S., Crema, E., Masino, L., Vecli, A., Viappiani, C., Small, J. R., Libertini, L. J., and Small, E. W. (2000) Fast events in protein folding. Structural volume changes accompanying the early events in the $\mathrm{N} \rightarrow \mathrm{I}$ transition of apomyoglobin induced by ultrafast pH jump, Biophys. J. 78, 405-415.

14. Miksovska, J., and Larsen, R. W. (2003) Photothermal studies of $\mathrm{pH}$ induced unfolding of apomyoglobin, Protein J. 22, 387-394.

15. Dutta, A. K., Kamada, K., and Ohta, K. (1996) Spectroscopic studies of Nile red in organic solvents and polymers, J. Photochem. Photobiol., A 93, 57-64.

16. Ghoneim, N. (2000) Photophysics of Nile red in solution steady state spectroscopy, Spectrochim. Acta, Part A 56, 1003-1010.

17. Golini, C. M., Williams, B. W., and Foresman, J. B. (1998) Further solvatochromic, thermochromic and theoretical studies on Nile red, J. Fluoresc. 8, 395-404.

18. Hou, Y., Bardo, A. M., Martinez, C., and Higgins, D. A. (2000) Characterization of molecular scale environments in polymer films by single molecule spectroscopy, J. Phys. Chem. B 104, 212219.

19. Sackett, D. L., and Wolff, J. (1987) Nile red as a polarity-sensitive fluorescent probe of hydrophobic protein surfaces, Anal. Biochem. $167,228-234$.

20. Hendriks, J., Gensch, T., Hviid, L., Horst, M. A. v. d., Hellingwerf, K. J., and Thor, J. J. v. (2002) Transient exposure of hydrophobic surface in the photoactive yellow protein monitored with Nile red, Biophys. J. 82, 1632-1643.

21. Tirado Rives, J., and Jorgensen, W. L. (1993) Molecular dynamics simulations of the unfolding of apomyoglobin in water, Biochemistry 32, 4175-4184.

22. Rossi Fanelli, A., Antonini, E., and Caputo, A. (1958) Studies on the structure of hemoglobin. I. Physicochemical properties of human globin, Biochim. Biophys. Acta 30, 608-615.

23. Harrison, S. C., and Blout, R. (1965) Reversible conformational changes of myoglobin and apomyoglobin, J. Biol. Chem. 240, 299-303.

24. Marshall, A. G. (1978) Biophysical Chemistry: Principles, Techniques and Applications, John Wiley and Sons, New York.

25. Bonetti, G., Vecli, A., and Viappiani, C. (1997) Reaction volume of water formation detected by time-resolved photoacoustics: photoinduced proton transfer between $o$-nitrobenzaldehyde and hydroxyls in water, Chem. Phys. Lett. 269, 268-273.

26. Viappiani, C., Bonetti, G., Carcelli, M., Ferrari, F., and Sternieri, A. (1998) Study of proton-transfer processes in solution using the laser induced $\mathrm{pH}$-jump: a new experimental setup and an improved data analysis based on genetic algorithms, Rev. Sci. Instrum. 69, 270-276.
27. George, M. V., and Scaiano, J. C. (1980) Photochemistry of o-nitrobenzaldehyde and related studies, J. Phys. Chem. 84, 492495.

28. Carcelli, M., Pelagatti, P., and Viappiani, C. (1998) Determination of the $\mathrm{p} K_{\mathrm{a}}$ of the aci-nitro intermediate in $o$-nitrobenzyl systems, Isr. J. Chem. 38, 213-221.

29. Abbruzzetti, S., Carcelli, M., Rogolino, D., and Viappiani, C. (2003) Deprotonation yields, $\mathrm{p} K_{\mathrm{a}}$, and aci-nitro decay rates in some substituted o-nitrobenzaldehydes, Photochem. Photobiol. Sci. 2, 796-800.

30. Abbruzzetti, S., Viappiani, C., Small, J. R., Libertini, L. J., and Small, E. W. (2001) Kinetics of histidine deligation from the heme in $\mathrm{GuHCl}$ unfolded $\mathrm{Fe}(\mathrm{III})$ cytochrome $c$ studied by a laser induced pH-jump technique, J. Am. Chem. Soc. 123, 6649-6653.

31. Abbruzzetti, S., Viappiani, C., Sinibaldi, F., and Santucci, R. (2004) Kinetics of histidine deligation from the heme Fe(III) in Nfragment (residues 1-56) of cytochrome c, Protein J. 23, 519527.

32. Gutman, M., Huppert, D., and Pines, E. (1981) The pH Jump: A rapid modulation of $\mathrm{pH}$ of aqueous solution by a laser pulse, $J$. Am. Chem. Soc. 103, 3709-3713.

33. Abbruzzetti, S., Sottini, S., Viappiani, C., and Corrie, J. E. T. (2005) Acid-induced unfolding of myoglobin triggered by a laser pH-jump method, Photochem. Photobiol. Sci. in press.

34. Abbruzzetti, S., Grandi, E., Viappiani, C., Bologna, S., Campanini, B., Raboni, S., Bettati, S., and Mozzarelli, A. (2005) Kinetics of Acid-Induced Spectral Changes in the GFPmut2 Chromophore, J. Am. Chem. Soc. 127, 626-635.

35. Wagner, P. J., and Siebert, E. J. (1981) Deactivation of triplet phenyl alkyl ketones by conjugatively electron-withdrawing substituents, J. Am. Chem. Soc. 103, 7329-7335.

36. Wagner, U. G., Muller, N., Schmitzberger, W., Falk, H., and Kratky, C. (1995) Structure determination of the biliverdin apomyoglobin complex: crystal structure analysis of two crystal forms at 1.4 and 1.5 A resolution, J. Mol. Biol. 247, 326-337.

37. Cocco, M., Kao, Y. H., Phillips, A., and Lecomte, J. T. J. (1992) Structural comparison of apomyoglobin and metaquomyoglobin: $\mathrm{pH}$ titration of histidines by NMR spectroscopy, Biochemistry 31, 6481-6491.

38. Mehler, E. L., and Guarnieri, F. (1996) Self-consistent, free energy based approximation to calculate $\mathrm{pH}$ dependent electrostatic effects in proteins, J. Phys. Chem. 100, 16006-16018.

39. Mehler, E. L., and Guarnieri, F. (1999) A self-consistent, microenvironment modulated screened Coulomb potential approximation to calculate $\mathrm{pH}$-dependent electrostatic effects in proteins, Biophys. J. 77, 3-22.

40. Caflisch, A., and Karplus, M. (1995) Acid and thermal denaturation of barnase investigated by molecular dynamics simulations, J. Mol. Biol. 252, 672-708.

41. Ponder, J. W., and Richards, F. M. (1987) An efficient Newtonlike method for molecular mechanics energy minimization of large molecules, J. Comput. Chem. 8, 1016-1024.

42. Brooks, B. R., Bruccoleri, R. E., Olafson, B. D., States, D. J., Swaminathan, S., and Karplus, M. (1983) CHARMM: a program for macromolecular energy minimization and dynamic calculations, J. Comput. Chem. 4, 187-217.

43. Bashford, D., and Case, D. A. (2000) Generalized born models of macromolecular solvation effects, Annu. Rev. Phys. Chem. 51, 129-152.

44. Feig, M., and Brooks, C. L. (2002) Evaluating CASP4 predictions with physical energy functions, Proteins 49, 232-245.

45. Qiu, D., Shenkin, P. S., Hollinger, F. P., and Still, W. C. (1997) The GB/SA continuum model for solvation. A fast analitical method for the calculation of approximate Born radii, J. Phys. Chem. A 101, 3005-3014.

46. Koradi, R., Billeter, M., and Wuthrich, K. (1996) MOLMOL: a program for display and analysis of macromolecular structures, J. Mol. Graphics 14, 51-55.

47. Kabsch, W., and Sander, C. (1983) Dictionary of protein secondary structure: pattern recognition of hydrogen-bonded and geometrical features, Biopolymers 22, 51-55.

48. Binkowski, T. A., Naghibzadeh, S., and Liang, J. (2003) CASTp: Computed Atlas of Surface Topography of proteins, Nucleic Acids Res. 31, 3352-3355.

49. Liang, J., Edelsbrunner, H., and Woodward, C. (1998) Anatomy of protein pockets and cavities: measurement of binding site geometry and implications for ligand design, Protein Sci. 7, 18841897. 
50. Humphrey, W., Dalke, A., and Schulten, K. (1996) VMD: visual molecular dynamics, J. Mol. Graphics 14, 33-38.

51. Morris, G. M., Goodsell, D. S., Halliday, R. S., Huey, R., Hart, W. E., Belew, R. K., and Olson, A. J. (1998) Automated docking using a Lamarckian genetic algorithm and an empirical binding free energy function, J. Comput. Chem. 19, 1639-1662.

52. Gasteiger, J., and Marsili, M. (1980) Iterative partial equalization of orbital electronegativity-a rapid access to atomic charges, Tetrahedron 36, 3219-3228.

53. Weiner, S. J., Kollman, P. A., Case, D. A., Singh, U. C., Alagona, G. C., Profeta, S., and Weiner, P. (1984) A new force field for molecular mechanical simulation of nucleic acids and proteins, J. Am. Chem. Soc. 106, 765-784.

54. Bismuto, E., Gratton, E., and Lamb, D. C. (2001) Dynamics of ANS binding to tuna apomyoglobin measured with fluorescence correlation spectroscopy, Biophys. J. 81, 3510-3521.

55. Gutman, M., Nachliel, E., and Huppert, D. (1982) Direct measurement of proton transfer as a probing reaction for the microenvironment of the apomyoglobin heme-binding site, Eur. J. Biochem. $125,175-181$.

56. Shimoni, E., Nachliel, E., and Gutman, M. (1993) Gaugement of the inner space of the apomyoglobin's heme binding site by a single free diffusing proton. II. Interaction with a bulk proton, Biophys. J. 64, 480-483.

57. Shimoni, E., Tsfadia, Y., Nachliel, E., and Gutman, M. (1993) Gaugement of the inner space of the apomyoglobin's heme binding site by a single free diffusing proton I. Proton in the cavity, Biophys. J. 64, 472-479.
58. Henry, E. R., and Hofrichter, J. (1992) in Numerical Computer Methods (Brand, L., and Johnson, M. L., Eds.) pp 129-192, Academic Press, Inc., San Diego, CA.

59. Eliezer, D., Yao, J., Dyson, H. J., and Wright, P. E. (1998) Structural and dynamic characterization of partially folded states of apomyoglobin and implications for protein folding, Nat. Struct. Biol. 5, 148-155.

60. Tcherkasskaya, O., and Ptitsyn, O. B. (1999) Molten globule versus variety of intermediates: influence of anions on $\mathrm{pH}$-denatured apomyoglobin, FEBS Lett. 455, 325-331.

61. Eliezer, D., Chung, J., Dyson, H. J., and Wright, P. E. (2000) Native and non-native secondary structure and dynamics in the pH 4 intermediate of apomyoglobin, Biochemistry 39, 2894-2901.

62. Uzawa, T., Akiyama, S., Kimura, T., Takahashi, S., Ishimori, K., Morishima, I., and Fujisawa, T. (2004) Collapse and search dynamics of apomyoglobin folding revealed by submillisecond observations of $\alpha$-helical content and compactness, Proc. Natl. Acad. Sci. U.S.A. 101, 1171-1176.

63. Nishimura, C., Dyson, H. J., and Wright, P. E. (2002) The apomyoglobin folding pathway revisited: structural heterogeneity in the kinetic burst phase intermediate, J. Mol. Biol. 322, 483489.

64. Rosenfield, R. J., Goodesell, D. S., Musah, R. A., Morris, G. M., Goodin, D. B., and Olson, A. J. (2003) Automated docking of ligands to an artificial active site: augmenting crystallographic analysis with computer modeling, J. Comput.-Aided Mol. Des. $17,525-536$.

BI051905Y 